Swarthmore College

Works

3-1-1998

\title{
A Decade Of Starspot Activity On The Eclipsing Short-Period RS Canum Venaticorum Star WY Cancri: 1988-1997
}

\author{
P. A. Heckert \\ G. V. Maloney \\ M. C. Stewart \\ J. I. Ordway \\ Mary Ann Hickman \\ Swarthmore College, mklasse1@swarthmore.edu
}

See next page for additional authors

Follow this and additional works at: https://works.swarthmore.edu/fac-physics

Part of the Astrophysics and Astronomy Commons

Let us know how access to these works benefits you

\section{Recommended Citation}

P. A. Heckert, G. V. Maloney, M. C. Stewart, J. I. Ordway, Mary Ann Hickman, and M. Zeilik. (1998). "A Decade Of Starspot Activity On The Eclipsing Short-Period RS Canum Venaticorum Star WY Cancri: 1988-1997". Astronomical Journal. Volume 115, Issue 3. 1145-1152. DOI: 10.1086/300238 https://works.swarthmore.edu/fac-physics/213

This work is brought to you for free by Swarthmore College Libraries' Works. It has been accepted for inclusion in Physics \& Astronomy Faculty Works by an authorized administrator of Works. For more information, please contact myworks@swarthmore.edu. 


\section{Authors}

P. A. Heckert, G. V. Maloney, M. C. Stewart, J. I. Ordway, Mary Ann Hickman, and M. Zeilik 
The Astronomical Journal, 115:1145-1152, 1998 March

(C) 1998. The American Astronomical Society. All rights reserved. Printed in U.S.A.

\title{
A DECADE OF STARSPOT ACTIVITY ON THE ECLIPSING SHORT-PERIOD RS CANUM VENATICORUM STAR WY CANCRI: 1988-1997
}

\author{
Paul A. Heckert, George V. Maloney, Maria C. Stewart, and James I. Ordway \\ Department of Chemistry and Physics, Western Carolina University, Cullowhee, NC 28723; heckert@wcu.edu \\ MARY ANN HiCKMAN \\ Department of Physics and Astronomy, Agnes Scott College; and Department of Physics and Astronomy, \\ Swarthmore College, Swarthmore, PA 19801-1397; mhickma1@swarthmore.edu \\ AND \\ MiCHAEL ZEILIK \\ Institute for Astrophysics, University of New Mexico, Albuquerque, NM 87131; zeilik@chicoma.la.unm.edu \\ Received 1997 July 14 ; revised 1997 November 24
}

\begin{abstract}
We present optical photometry of the short-period eclipsing RS CVn system WY Cancri for the years 1988-1997. For each light curve, we model the distortion waves in order to study the behavior of starspots in this system. After removing the spot effects from the light curves, we model the cleaned data to obtain system parameters. We also note changes in the luminosity of the primary star that are not explained by the spot variation.

Key words: binaries: eclipsing — stars: activity — stars: individual (WY Cancri) — stars: spots
\end{abstract}

\section{INTRODUCTION}

WY Cancri is a member of the short-period eclipsing group of RS CVn stars defined by Hall (1976). It is a singlelined spectroscopic binary with spectral types G5 V and approximately M2. As part of an ongoing study of this group, Zeilik et al. (1990) used the information limit optimization technique (ILOT) of Budding \& Zeilik (1987) to analyze the available photometry of WY Cnc from 1964 to 1989. They also reviewed the pertinent literature on this system. Rao, Sarma, \& Rao (1991) also applied similar techniques to model data from five epochs between 1973 and 1986.

Unfortunately, for most of that time period, WY Cnc was observed sporadically. It is therefore difficult to draw any conclusions about the nature of starspot cycles beyond the trend observed by Zeilik et al. (1990): the spots occur in the active longitude belts at roughly $90^{\circ}$ and $270^{\circ}$. In 1988 , we therefore began a program to collect systematic annual light curves of WY Cnc. The 1988 and $1989 B$ and $V$ light curves and models are reported in Heckert \& Zeilik (1990) and included in the analysis of Zeilik et al. (1990). In this paper, we include them and report new $B V R I$ light curves for 1990-1997.

\section{OBSERVATIONS AND DATA}

We conducted all observations using the Mount Laguna Observatory 24 inch $(61 \mathrm{~cm})$ telescope, operated by San Diego State University. A complete log of observation dates is in Table 1 . The previously reported 1988 and 1989 data, as well as the 1990 January data, used an EMI 6256 phototube operated at $-1300 \mathrm{~V}$ and equipped with $B$ and $V$ filters designed to closely match the Johnson $U B V$ system. The EMI tube was subsequently replaced with a redsensitive Hamamatsu R943-02 GaAs tube. Hence, the observations from 1990 May to 1997 used this tube operated at $-1450 \mathrm{~V}$ and equipped with $B V R I$ filters designed to closely match the Johnson-Cousins system. Each observation consisted of two separate $40 \mathrm{~s}$ integrations with the EMI tube or two separate $20 \mathrm{~s}$ integrations with the more sensitive Hamamatsu tube. These integrations were typically long enough to produce a few hundred thousand counts and, therefore, photon statistics well under $1 \%$. We only worked on nights when the atmospheric conditions were sufficient to produce statistical errors of less than $1 \%$ (0.01 mag). We used either a $19^{\prime \prime}$ or a $26^{\prime \prime}$ aperture as dictated by the seeing conditions and the Moon's brightness. SAO 80583 was our comparison star for all the observations, SAO 80598 the check star. The calibrated magnitudes for SAO 80583 are $B=8.60, V=8.31, R=8.14$, and $I=7.99$. All data are transformed into differential magnitudes in the standard Johnson-Cousins system.

We plot our new data from the years 1990-1997 in Figures 1-14. The data are also archived in the IAU Commission 27 data archives (file 322E; see Schmidt 1992). We were able to obtain the most complete light curves during 1991, 1994, and 1995. We are missing only the first half of the primary eclipse during 1990, the beginning of the secondary eclipse during 1997, and parts of both the primary and secondary eclipses during 1996. The year 1992 marked the end of a major drought in California, so our 1992 light curves have significant gaps. Hence, the models for that year should be viewed with a healthy skepticism. In 1993, we were unable to obtain a complete light curve. The 1993 data are archived with the other data but are not used in this paper.

\section{ANALYSIS AND RESULTS}

In Figure 15, we superpose the $V$ data for all years from 1988 to 1997 . Note that during 1988 and again in 1997, WY Cnc is significantly brighter during the out-of-eclipse phases and the secondary eclipse than in other years. This change cannot be caused by a variable comparison star, or a calibration error, because the agreement during primary eclipse is good for all years. Sarma (1976) found similar long-term variations comparing light curves from 1965, 1969, 1970, and 1974 and claims a roughly 10 year period for these variations. He refers to them as secular variations. Rao et al. (1991) also report similar secular variations for light curves between 1973 and 1986. They claim a 50 year period. More 
TABLE 1

LOG OF OBSERVATIONS

\begin{tabular}{|c|c|c|}
\hline Year & Nights of Observation & Filter \\
\hline 1988. & May $10-15,19-21$ & $B V$ \\
\hline $1989 \ldots \ldots$ & Mar 8-10; May 8, 13, 18, 19 & $B V$ \\
\hline $1990 \ldots \ldots$ & $\begin{array}{l}\text { Jan } 1,4,5,7,8 ; \text { May } 13-18,25 \\
\quad 26,30\end{array}$ & $B V$ Jan; $B V R I$ May \\
\hline $1991 \ldots \ldots$ & $\begin{array}{c}\text { Jan } 9,11,13,14 ; \text { Mar } 3,7,9 ; \\
\quad \text { May } 12-17,19,22-24,26\end{array}$ & $B V R I$ \\
\hline $1992 \ldots \ldots$ & May $12,13,15,17,18,20,26,27$ & $B V R I$ \\
\hline $1993 \ldots \ldots$ & Jan $1,2,4$ & $B V R I$ \\
\hline $1994 \ldots \ldots$ & Jan $4,6,7,10$ & $B V R I$ \\
\hline $1995 \ldots \ldots$ & Feb 2, 3, 5, 6, 9, 18, 19 & $B V R I$ \\
\hline $1996 \ldots \ldots$ & 1995 Dec 28; Jan 3, 5, 8 & $B V R I$ \\
\hline $1997 \ldots \ldots$ & $\operatorname{Jan} 7,8,10,11$ & $B V R I$ \\
\hline
\end{tabular}

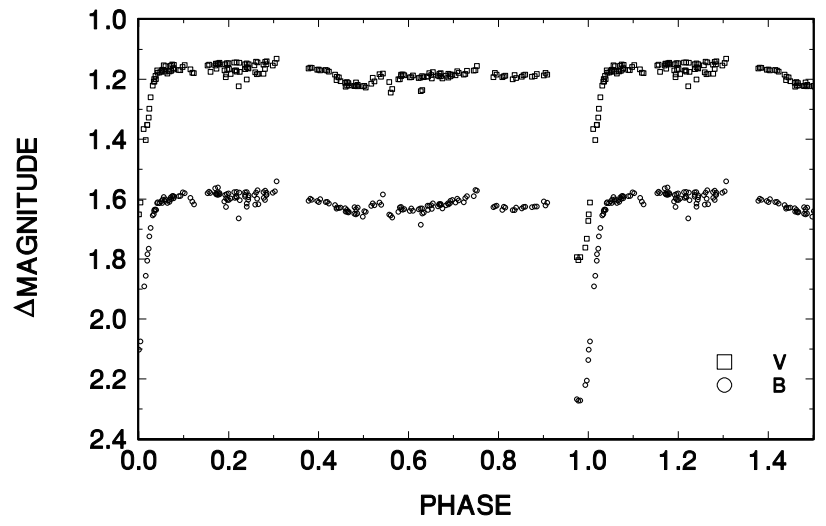

FIG. 1.-Differential $B$ and $V$ magnitudes for WY Cnc during 1990

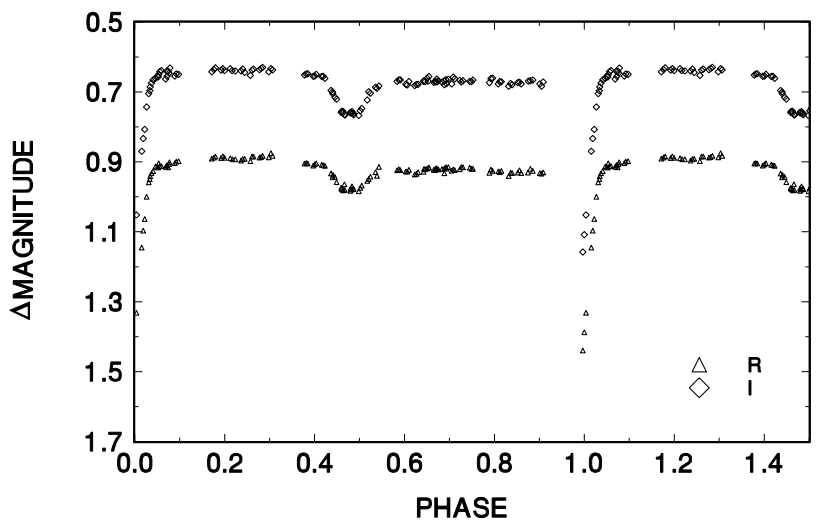

FIG. 2.-Differential $R$ and $I$ magnitudes for WY Cnc during 1990

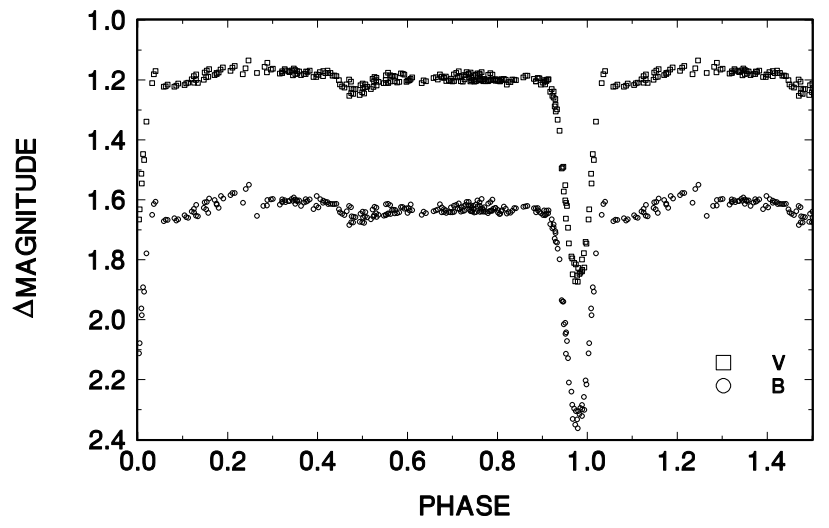

FIG. 3.-Differential $B$ and $V$ magnitudes for WY Cnc during 1991

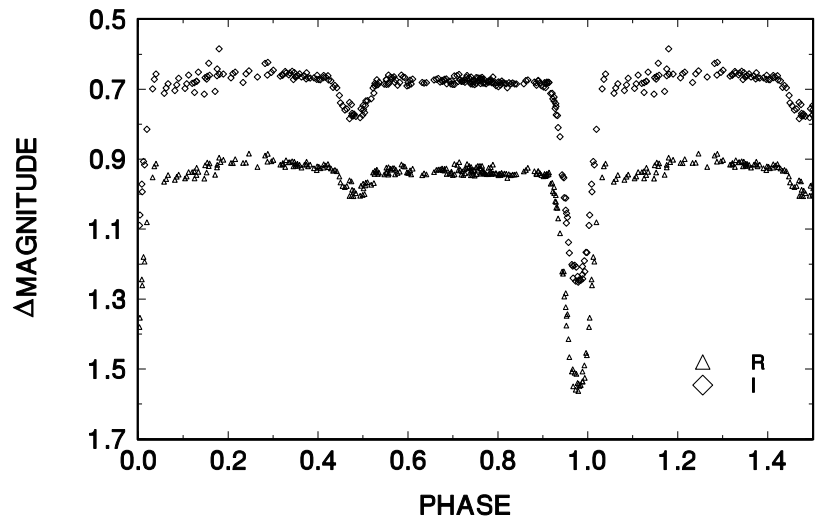

FIG. 4.-Differential $R$ and $I$ magnitudes for WY Cnc during 1991

notably, their 1985-1986 light curves are brightest, suggesting that the bright phase lasts a few years. Our data are consistent with a roughly 10 year period but are insufficient to either prove or refute these period claims. What causes these secular variations? We suggest two possibilities: polar or longitudinally distributed spots and pulsations.

On the basis of Doppler imaging, polar spots have long been suggested as occurring on RS CVn stars (Hatzes et al. 1996 and references therein). Rao et al. (1991) independently suggested either polar or longitudinally distributed spots to explain this effect in WY Cnc. The ILOT is only sensitive to

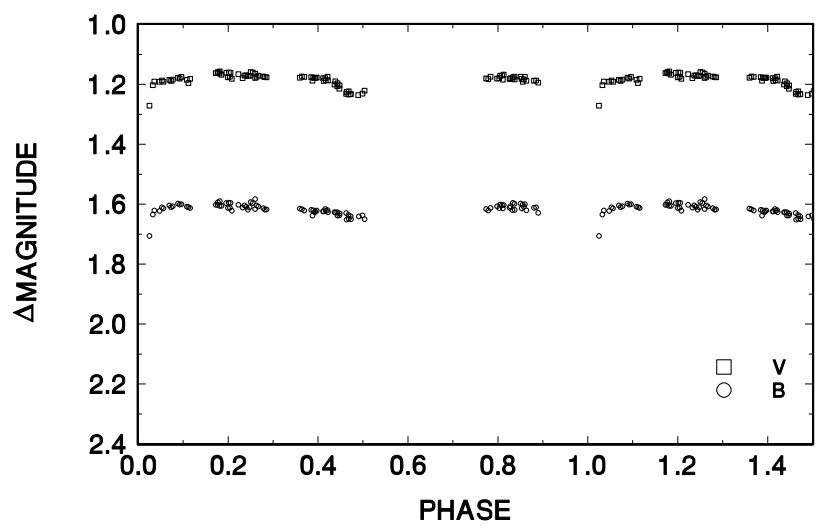

Fig. 5.-Differential $B$ and $V$ magnitudes for WY Cnc during 1992

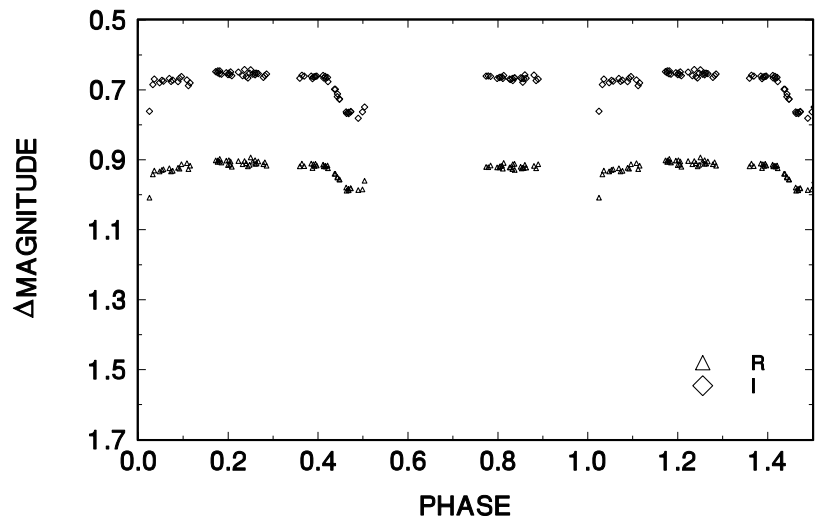

FIG. 6.-Differential $R$ and $I$ magnitudes for WY Cnc during 1992 


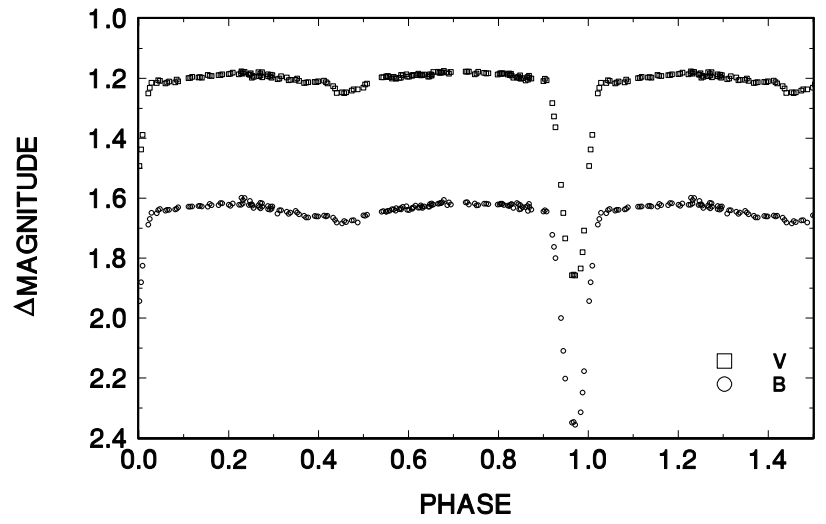

FIG. 7.-Differential $B$ and $V$ magnitudes for WY Cnc during 1994

spots that are concentrated into one or two groups, not to symmetric polar spots or to spots longitudinally distributed over the star. If the primary star has either polar or longitudinally distributed spots that disappeared during 1988 and 1997, the out-of-eclipse and secondary eclipse parts of the light curve would be brighter during these years. The primary eclipse would be unaffected. If this hypothesis is correct, then WY Cnc normally has either a polar spot or longitudinally distributed spots in addition to the large spot

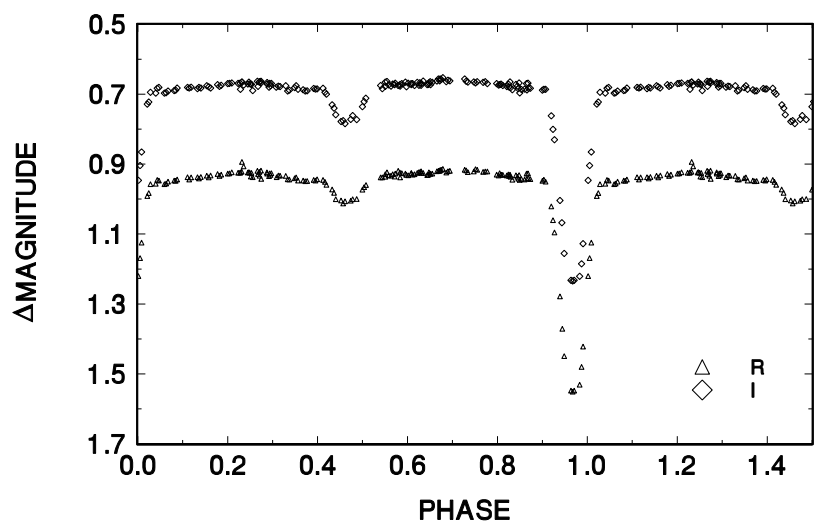

FIG. 8.-Differential $R$ and $I$ magnitudes for WY Cnc during 1994

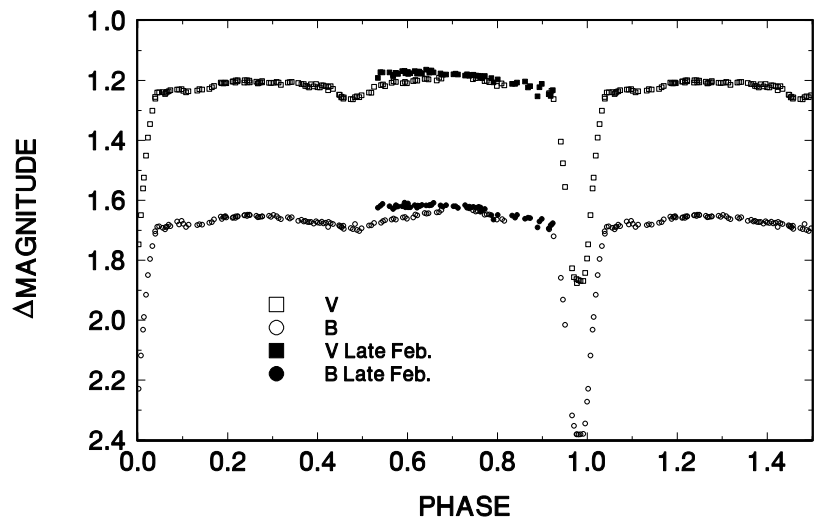

FIG. 9.-Differential $B$ and $V$ magnitudes for WY Cnc during 1995. As discussed in the text, the data between phases $\sim 0.5$ and $\sim 0.9$ that are plotted with filled symbols were taken about 10 days later than the other data and indicate a rapid change in the light curve. These data were not used in the models for 1995.

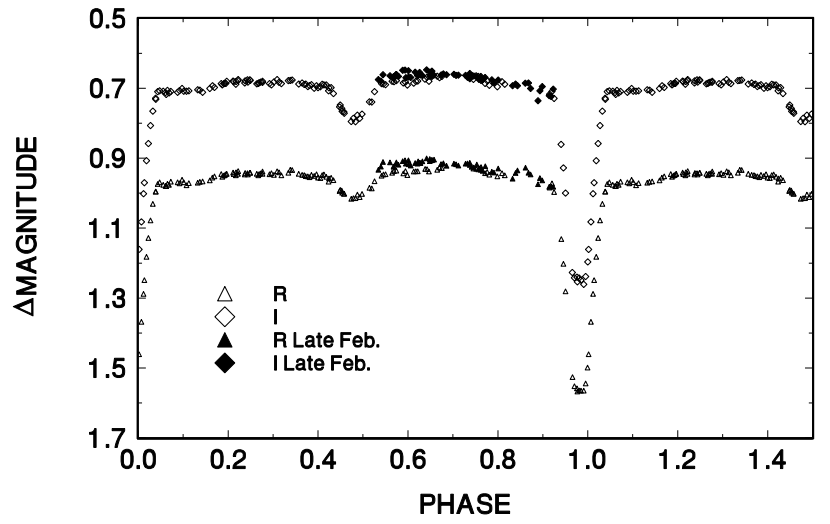

Fig. 10.-Same as Fig. 9, but for $R$ and $I$

groups we model. This spot (or spots) apparently dispersed during 1988 and again during 1997.

We also note a rapid but smaller similar brightening during 1995. Figures 9 and 10 show our 1995 light curves. During 1995, we observed WY Cnc between February 2 and 9. At this point we had a nearly complete light curve, with small gaps at phases $\sim 0.7$ and $\sim 0.9$. The weather did not permit us to fill in the gaps until February 18 and 19, roughly 10 days later. As shown in Figures 9 and 10, there is a similar but smaller variation for the 1995 February 18 and 19 observations. Unfortunately, we do not have a complete light curve after this change. In the context of this model,

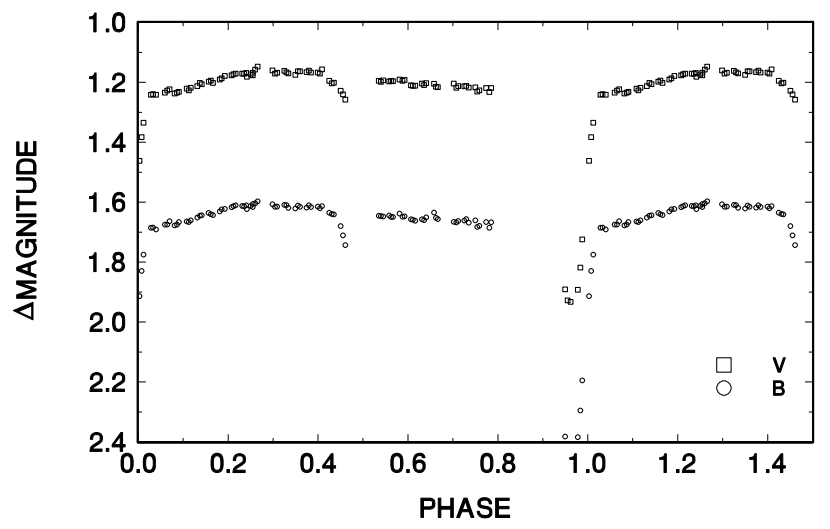

FIG. 11.-Differential $B$ and $V$ magnitudes for WY Cnc during 1996

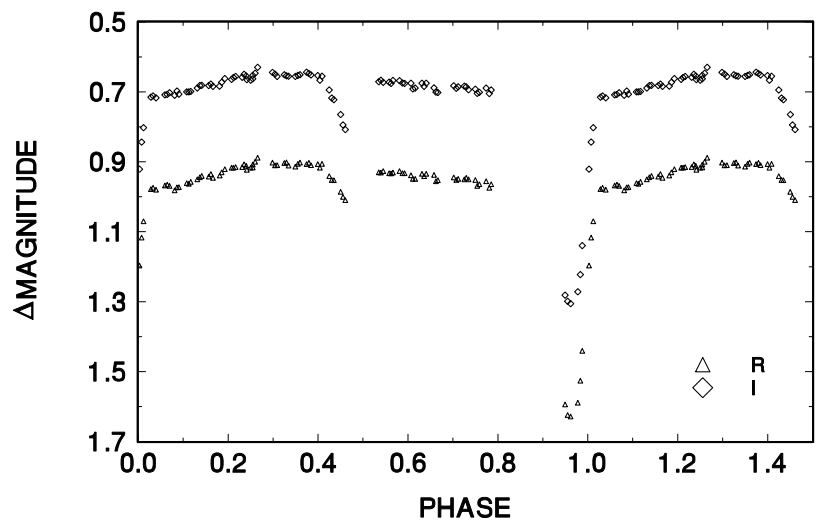

FIG. 12.-Differential $R$ and $I$ magnitudes for WY Cnc during 1996 


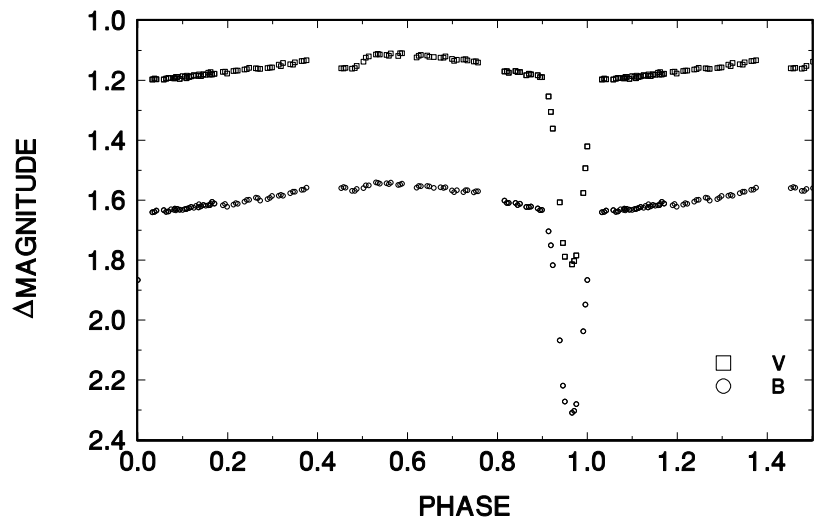

FIG. 13. - Differential $B$ and $V$ magnitudes for WY Cnc during 1997

either a polar spot dispersed rapidly or longitudinally distributed spots coalesced into one spot or disappeared simultaneously. The brighter stage was short-lived; by 1996, WY Cnc was at its more typical lower brightness. This model requires that the polar spot or longitudinally distributed spots remain very stable for several years and then disperse rather rapidly. The rapid dispersion is quite likely in such a complex system, but a decade of stability coupled with the rapid dispersion seems less so.

To explain the observed brightness decrease of roughly $0.08-0.1 \mathrm{mag}$ with a dark $(0 \mathrm{~K})$ spot requires that $8 \%$ to

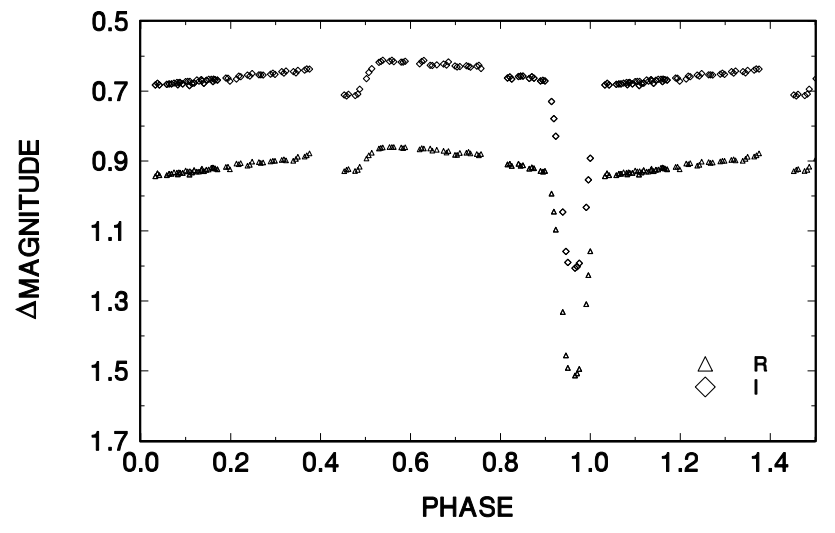

FIG. 14.-Differential $R$ and $I$ magnitudes for WY Cnc during 1997

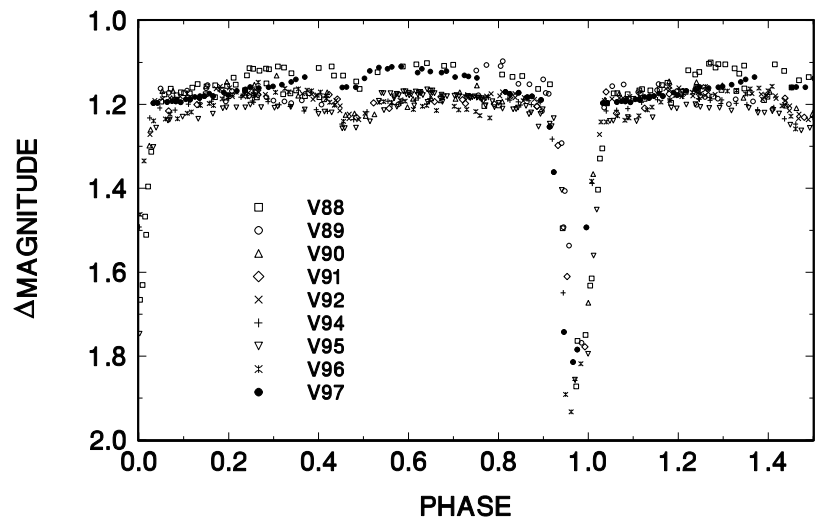

FIG. 15.-Differential $V$ magnitudes for WY Cnc for the years from 1988 to 1997 . Note the increased brightness during 1988 and 1997 outside the primary eclipse, discussed in the text. To reduce crowding of the plotted points only every $n$th point is plotted, where $n$ is chosen for each year.
$10 \%$ of the star's area, as projected onto the observer's line of sight, be covered by the spot. Because the projected area is affected by the cosine of the latitude factor and WY Cnc has a $90^{\circ}$ inclination, a polar spot symmetrically centered at $90^{\circ}$ latitude would have to be fairly large to produce the required $8 \%-10 \%$ projected area. In simple terms, the pole is not visible from Earth. A series of longitudinally distributed spots, approximated by a dark equatorial band, would not need to be as large.

It is instructive to compute the size spot required for each case. To do so, one can integrate the area element on the surface as projected onto the plane of the observer and then divide by the total area projected on the same plane. Assuming a completely dark spot, ignoring complicating factors such as limb darkening and nonspherical effects, and integrating over the appropriate latitudes, we find the minimum latitude range needed. A dark equatorial band with no gaps would have to extend about $4^{\circ}$ in latitude both above and below the equator to explain a $10 \%$ brightness decrease. A series of spots evenly distributed longitudinally would have gaps and hence extend a little farther above or below the equator. A polar spot would need to extend from the pole to about $45^{\circ}$ latitude to explain a $10 \%$ brightness decrease. Of course, real spots would not be completely dark and must therefore be larger. While not being physically impossible, a polar spot of this size leads all but the most devoted aficionados of polar spots to seek an alternative explanation.

Could the primary star be pulsating? Note that during both 1988 and 1997 our clean fits yield both larger than average values for the radius of the primary star and lower than average values for the ratio of the radii $\left(k=r_{2} / r_{1}\right)$, as would be expected if the luminosity variations were pulsations (see Table 3 below). To further test this possibility, we computed the values of $R_{1}$ in solar units from the average values of the clean fit parameters for each year. Following the method outlined later in this paper, we obtain for the radius of the primary star $0.98 \pm 0.07,0.91 \pm 0.03$, $0.93 \pm 0.02, \quad 0.86 \pm 0.06, \quad 0.95 \pm 0.02, \quad 0.96 \pm 0.04$, $0.95 \pm 0.02$, and $0.96 \pm 0.02 R_{\odot}$ for the years 1988,1990 , 1991, 1992, 1994, 1995, 1996, and 1997, respectively (see Fig. 16). The errors are from the standard deviations of the values for the different colors for each year. Note that the highest values of $R_{1}$ occur during 1988, 1995, and 1997.

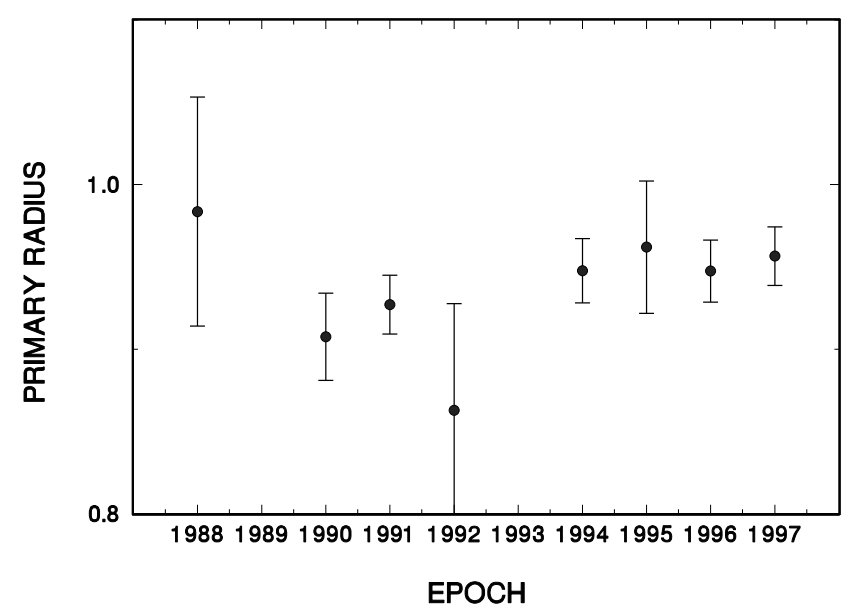

FIG. 16.-Radius of the primary star in solar radii computed from the model fits for each year. 
During 1988 and 1997 the primary was bright; during 1995, the primary apparently brightened (although not as much) within 2 weeks of the time the modeled data were taken. The errors are large enough to make definite conclusions difficult, but it is a reasonable possibility that the secular variations in the primary could be some type of pulsation. We reject the possibility that episodes of mass loss produce, for a brief period, a circumstellar shell that mimics the pulsation, because Rao et al. (1991) detected no period changes between 1931 and 1986.

The modeled changes in $R_{1}$ are about the right amount to explain the observed brightness difference of $\sim 0.08-0.1$ mag during the secondary eclipse. Because the errors in $R_{1}$ for a single year are large, we compare the average values for the bright years (1988 and 1997) and for the other years. For 1988 and 1997, the average modeled value of $R_{1}$ is 0.97 $R_{\odot}$. For the other years it is $0.93 R_{\odot}$. This $4 \%$ change in radius will produce an $8 \%$ change in the star's surface area and a corresponding $8 \%$ change in the star's brightness, if the temperature does not change.

To investigate temperature changes in the primary star, we computed the $B-V, V-R$, and $V-I$ color indexes at the middle of the secondary eclipse. The 1989 and 1996 data were excluded because of gaps in the light curves during the secondary eclipse. The year-to-year differences in these color indexes were comparable to the level expected with $1 \%$ errors in the photometry. The range from highest to lowest value was 0.69 to 0.73 for $B-V, 0.40$ to 0.42 for $V-R$, and 0.77 to 0.79 for $V-I$. We also note that for the 1992 data the $B-V$ index indicates a warmer than average temperature, while the other indexes indicate a cooler than average temperature. With that caveat, however, we note that the color indexes indicate warmer temperatures when the star is brightest, in 1988 and 1997, as observed in Cepheid variables. For the $B-V$ color index, the average of the 1988 and 1997 temperatures is $80 \mathrm{~K}$ warmer than the average for the other years. We have no $V-R$ or $V-I$ color indexes for 1988, but using these indexes for 1997 yields slightly smaller temperature differences.

It is also useful to estimate the expected temperature change. By applying the Stefan-Boltzmann law and taking the ratios of the luminosities, we find that a luminosity change of about $10 \%$ with a radius change from 0.97 to 0.93 $R_{\odot}$ will correspond to a temperature change of only about $15 \mathrm{~K}$. Values of the maximum and minimum radii differing by $\sim 1 \%$ from these values will produce correspondingly different values in the required temperature change, which can then be as high as $\sim 70 \mathrm{~K}$. We again stress that the possible temperature changes are near the limit of the accuracy of our photometry. Hence, to within the accuracy of our data and models one cannot use observed temperatures, or their lack, to rule out pulsations to explain the observed secular variations.

We usually associate pulsations with post-mainsequence helium-burning evolutionary stages. Why would a main-sequence G star pulsate? Warner (1988) and Thomas (1979) suggested that solar-type stars can pulsate as a result of magnetic flux variations in the star's convective zone. If magnetic flux variations do indeed cause the observed secular variations, then they would be related to the magnetic activity cycle for WY Cnc. The magnetic cycle period would then follow the period of these secular variations, presumably about a decade (Maceroni et al. 1990). To conclusively observe manifestations of this magnetic cycle as starspot activity would therefore require systematic observations over a few decades.

The idea that the luminosity of the primary star varies by some mechanism related to magnetic cycles leads to an interesting speculation. Some evidence suggests such behavior in the Sun; during the Maunder minimum, there were very few sunspots and an apparent decrease in the Sun's luminosity. Could there be a similar relationship between luminosity and magnetic cycles on WY Cnc? If so, understanding these secular variations on WY Cnc will provide clues to understanding the relationship between the Sun's magnetic activity and long-term climatic cycles on Earth.

We analyze the starspot parameters using the ILOT of Budding \& Zeilik (1987). Zeilik et al. (1990) applied this technique to the data on WY Cnc available through 1989. This technique and its application to WY Cnc are described in detail in the above papers. The basic procedure is to fit the data to the eclipsing binary parameters, remove the eclipse effects and fit the data to the spot parameters, and then remove the spot effects and fit the cleaned light curve for the clean binary parameters. Our initial system parameters are from these papers.

The latitude is the most difficult spot parameter to fit with photometric modeling. As described by Budding \& Zeilik (1987), the ILOT estimates formal errors for determinant solutions and does not allow one to fit more parameters beyond what will produce a determinant solution. If a latitude error is reported, the ILOT was able to fit the data with a determinant latitude solution. Otherwise we fixed the latitude at a value to which it seemed to be converging in trial fits. Also note that because WY Cnc has a $90^{\circ}$ inclination, there is a north-south latitude ambiguity. Only positive latitudes are reported, but the spots could be in either hemisphere.

The modeling results are listed in Tables 2 and 3. In Table $2, \lambda, \beta$, and $\gamma$ are the longitude, latitude, and radius of spots 1 and 2 , respectively. The longitude is defined so that $0^{\circ}$ corresponds to a line from the primary to the secondary star (the center of the primary eclipse) and the longitude increases with phase. In Table $3, L_{1}$ and $L_{2}$ are the fractional luminosities of the primary and secondary stars. They are normalized to sum to approximately but not exactly one. The radius of the primary star, $r_{1}$, is expressed as a fraction of the semimajor axis. The ratio of the radii is $k=r_{2} / r_{1}$, and the mass ratio is $q=m_{2} / m_{1}$. The inclination, $i$, is in degrees. Figures 17 and 18 show initial, clean, and spot fits for the 1994 data.

Zeilik et al. (1990) found that the spots tend to occur in two active longitude belts (ALBs) roughly at quadrature, $90^{\circ}$ and $270^{\circ}$. They also noticed a trend toward low-latitude spots. With the new data, we still notice the tendency toward the $270^{\circ}$ ALB, as shown in Figure 19; however, the spots are not always well confined in the $90^{\circ}$ ALB. For example, we find a spot in 1991 at about $20^{\circ}-25^{\circ}$ longitude and in 1994 a spot at about $10^{\circ}-20^{\circ}$ longitude. This second spot was between $20^{\circ}$ and $40^{\circ}$ longitude during 1995 and 1996. It seems to have formed outside the ALB and then drifted toward it. We also note that both these spots are at low latitudes and that there seems to be a tendency for spots to drift toward increasing longitude within an ALB. We also find a spot in 1995 at about $150^{\circ}-155^{\circ}$ longitude. Apparently whatever is causing the ALB effect is confining spots to the $270^{\circ}$ ALB fairly well but not to the $90^{\circ}$ ALB. Why? 
TABLE 2

SPOT PARAMETERS

\begin{tabular}{|c|c|c|c|c|c|c|c|c|}
\hline Filter & $\lambda_{1}$ & $\beta_{1}$ & $\gamma_{1}$ & $\lambda_{2}$ & $\beta_{2}$ & $\gamma_{2}$ & $x^{2}$ & $N$ \\
\hline \multicolumn{9}{|l|}{ 1988: } \\
\hline$B \ldots \ldots$ & $35.7 \pm 4.8$ & $0.4 \pm 41.6$ & $6.9 \pm 0.4$ & $\ldots$ & $\ldots$ & $\ldots$ & 248.2 & 143 \\
\hline$V \ldots \ldots$ & $36.1 \pm 3.9$ & $9.7 \pm 40.0$ & $8.1 \pm 0.8$ & $\ldots$ & $\ldots$ & $\ldots$ & 253.0 & 147 \\
\hline \multicolumn{9}{|l|}{ 1989: } \\
\hline$B \ldots \ldots$ & $105 \pm 1.7$ & $5.2 \pm 37.6$ & $13.3 \pm 0.7$ & $\ldots$ & $\ldots$ & $\ldots$ & 684.6 & 179 \\
\hline$V \ldots \ldots$ & $116 \pm 3.4$ & 3.1 & 12.5 & $\ldots$ & $\ldots$ & $\ldots$ & 134.1 & 69 \\
\hline \multicolumn{9}{|l|}{ 1990: } \\
\hline B ... & $261.3 \pm 4.5$ & $82.2 \pm 2.1$ & $33.8 \pm 4.1$ & $\ldots$ & $\ldots$ & $\ldots$ & 150.7 & 137 \\
\hline$V \ldots \ldots$ & $255.9 \pm 4.4$ & $82.0 \pm 2.3$ & $32.9 \pm 4.5$ & $\ldots$ & $\ldots$ & $\ldots$ & 65.3 & 137 \\
\hline$R \ldots \ldots$ & $255.7 \pm 4.8$ & $83.5 \pm 2.1$ & $34.0 \pm 5.3$ & $\ldots$ & $\ldots$ & $\ldots$ & 47.3 & 137 \\
\hline$I \ldots \ldots$ & $242.0 \pm 4.9$ & $77.4 \pm 2.9$ & $24.7 \pm 3.2$ & $\ldots$ & $\ldots$ & $\ldots$ & 80.2 & 137 \\
\hline \multicolumn{9}{|l|}{ 1991: } \\
\hline$B \ldots \ldots$ & $242.6 \pm 3.4$ & $77.0 \pm 5.9$ & $28.3 \pm 7.1$ & $26.3 \pm 7.6$ & $0.0 \pm 13.8$ & $8.0 \pm 0.7$ & 540.2 & 280 \\
\hline$V \ldots \ldots$ & $246.1 \pm 4.7$ & $79.8 \pm 3.3$ & $27.2 \pm 4.6$ & $25.2 \pm 7.8$ & $0.0 \pm 18.5$ & $6.5 \pm 0.7$ & 377.3 & 280 \\
\hline$R \ldots \ldots$ & $245.1 \pm 6.9$ & $81.5 \pm 6.9$ & $27.5 \pm 11.8$ & $24.2 \pm 17.1$ & $0.0 \pm 21.1$ & $6.1 \pm 0.8$ & 306.5 & 280 \\
\hline$I \ldots$ & $239.2 \pm 7.8$ & $85.4 \pm 4.8$ & $35.5 \pm 17.7$ & $17.8 \pm 11.7$ & $0.0 \pm 26.3$ & $5.4 \pm 1.2$ & 312.2 & 280 \\
\hline \multicolumn{9}{|l|}{ 1992: } \\
\hline$B \ldots \ldots$ & $125.0 \pm 11.0$ & 45 (fixed) & $5.9 \pm 1.1$ & $\ldots$ & $\ldots$ & $\ldots$ & 53.9 & 96 \\
\hline$V \ldots \ldots$ & $239.9 \pm 31.6$ & 45 (fixed) & $5.1 \pm 3.3$ & $\ldots$ & $\ldots$ & $\ldots$ & 28.7 & 96 \\
\hline$R \ldots \ldots$ & $236.5 \pm 16.7$ & 45 (fixed) & $6.0 \pm 2.5$ & $\ldots$ & $\ldots$ & $\ldots$ & 27.2 & 96 \\
\hline$I \ldots \ldots$ & $224.5 \pm 25.7$ & 45 (fixed) & $4.8 \pm 3.1$ & $\ldots$ & $\ldots$ & $\ldots$ & 34.3 & 96 \\
\hline \multicolumn{9}{|l|}{ 1994: } \\
\hline$B \ldots \ldots$ & $133.5 \pm 8.3$ & $42.8 \pm 42.1$ & $7.1 \pm 4.2$ & $10.2 \pm 19.0$ & 0 (fixed) & $2.8 \pm 1.2$ & 76.8 & 174 \\
\hline$V \ldots \ldots$ & $139.8 \pm 9.7$ & 60 (fixed) & $9.7 \pm 1.1$ & $18.3 \pm 9.7$ & 45 (fixed) & $5.7 \pm 1.2$ & 47.9 & 174 \\
\hline$R \ldots \ldots$ & $144.0 \pm 9.8$ & 45 (fixed) & $7.3 \pm 0.9$ & $14.6 \pm 13.3$ & $0.0 \pm 41.8$ & $4.3 \pm 1.0$ & 56.4 & 174 \\
\hline$I \ldots \ldots$ & $144.0 \pm 10.9$ & 45 (fixed) & $7.6 \pm 0.9$ & $23.0 \pm 19.7$ & $0.0 \pm 50.1$ & $4.0 \pm 1.1$ & 58.0 & 174 \\
\hline \multicolumn{9}{|l|}{ 1995: } \\
\hline$B \ldots \ldots$ & $37.8 \pm 6.5$ & 45 (fixed) & $8.3 \pm 0.9$ & $152.3 \pm 9.1$ & 45 (fixed) & $7.0 \pm 1.0$ & 45.2 & 138 \\
\hline$V \ldots \ldots$ & $35.0 \pm 6.7$ & $40.5 \pm 34.9$ & $8.0 \pm 3.3$ & $152.5 \pm 10.6$ & $36.2 \pm 54.1$ & $6.0 \pm 3.4$ & 37.5 & 138 \\
\hline$R \ldots \ldots$ & $34.1 \pm 7.4$ & 45 (fixed) & $8.4 \pm 1.0$ & $153.4 \pm 11.5$ & 45 (fixed) & $6.6 \pm 1.2$ & 34.0 & 138 \\
\hline$I \ldots \ldots$ & $28.4 \pm 10.7$ & 45 (fixed) & $6.9 \pm 1.2$ & $154.7 \pm 11.4$ & $0.0 \pm 27.3$ & $4.7 \pm 1.1$ & 42.1 & 138 \\
\hline \multicolumn{9}{|l|}{ 1996: } \\
\hline$B \ldots \ldots$ & $258.6 \pm 4.7$ & $68.6 \pm 5.8$ & $24.7 \pm 4.3$ & $25.2 \pm 6.0$ & $0.0 \pm 31.6$ & $8.6 \pm 0.5$ & 171.9 & 93 \\
\hline$V \ldots \ldots$ & $254.8 \pm 4.5$ & 65 (fixed) & $21.4 \pm 0.8$ & $25.5 \pm 3.8$ & $0.0 \pm 21.0$ & $9.5 \pm 0.5$ & 104.3 & 93 \\
\hline$R \ldots \ldots$ & $255.2 \pm 5.5$ & $65.3 \pm 18.6$ & $19.8 \pm 9.6$ & $25.1 \pm 9.2$ & $2.4 \pm 18.6$ & $9.4 \pm 0.7$ & 85.0 & 93 \\
\hline$I \ldots \ldots$ & $250.7 \pm 6.0$ & $66.5 \pm 11.5$ & $19.1 \pm 6.0$ & $22.4 \pm 7.9$ & $0.0 \pm 25.6$ & $9.0 \pm 0.7$ & 68.0 & 93 \\
\hline \multicolumn{9}{|l|}{ 1997: } \\
\hline$B \ldots \ldots$ & $63.6 \pm 3.0$ & $44.1 \pm 37.2$ & $13.3 \pm 7.3$ & $317.3 \pm 7.5$ & $42.9 \pm 34.3$ & $8.7 \pm 4.3$ & 196.2 & 156 \\
\hline$V \ldots \ldots$ & $60.8 \pm 3.5$ & $43.9 \pm 30.2$ & $13.8 \pm 5.6$ & $318.0 \pm 6.6$ & $43.5 \pm 30.0$ & $10.0 \pm 3.9$ & 160.9 & 156 \\
\hline$R \ldots \ldots$ & $64.2 \pm 5.5$ & $43.7 \pm 19.8$ & $13.1 \pm 3.2$ & $327.5 \pm 9.8$ & $44.8 \pm 33.9$ & $9.8 \pm 4.3$ & 110.2 & 156 \\
\hline$I \ldots \ldots$ & $69.9 \pm 7.7$ & 45 (fixed) & $12.5 \pm 0.7$ & $334.4 \pm 13.2$ & 45 (fixed) & $9.1 \pm 0.9$ & 72.8 & 156 \\
\hline
\end{tabular}

What about latitude? Zeilik et al. (1990) noted a trend toward low-latitude spots. With our additional data, this trend seems to have been a statistical fluctuation caused by a small sample size. We note that in 1990 a spot appeared at $82^{\circ}$ latitude and remained at high latitude through 1991 . If WY Cnc has a polar spot, such a high-latitude spot could possibly be an appendage to the polar spot, but photo-

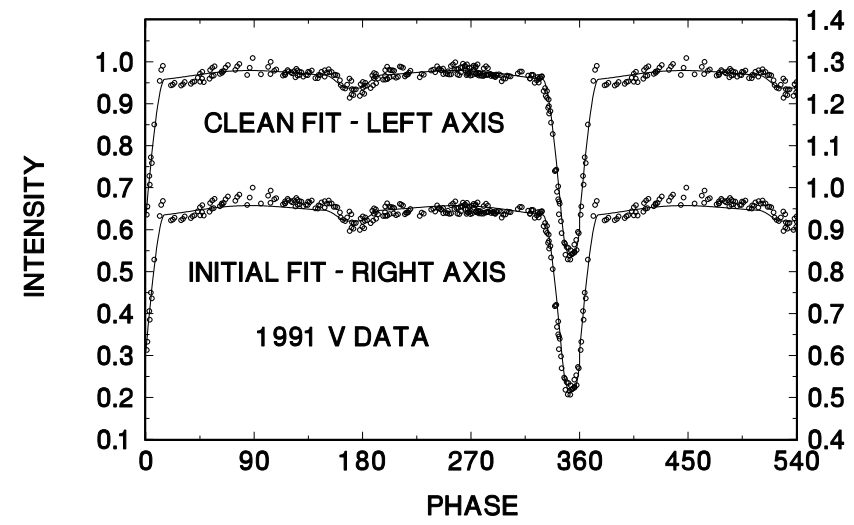

Fig. 17.- Initial and clean fits for the $1991 V$-band data. The lines represent the calculated models and the circles the data points. metric data do not allow us to be certain. (Note that during 1992 the latitude was indeterminate and fixed at $45^{\circ}$.) $\mathrm{We}$ also find a spot appearing at roughly $65^{\circ}$ latitude during 1996, and the 1997 spots are both at roughly $45^{\circ}$ latitude. Hence the full range of spot latitudes $\left(0^{\circ}-90^{\circ}\right)$ has been observed for WY Cnc. We also find that the spots in or near the $90^{\circ} \mathrm{ALB}$ tend to be at low latitudes and the spots in the

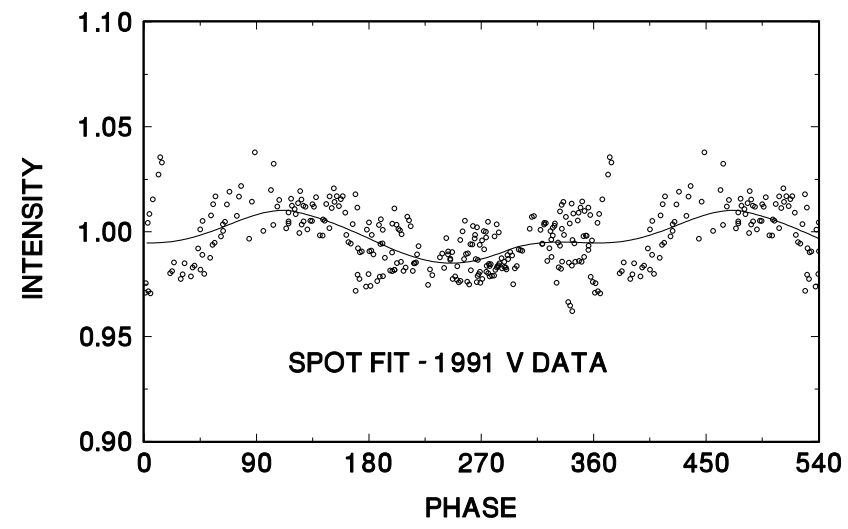

FIG. 18.-Spot fits for the $1991 V$-band data. The line represents the calculated model and the circles the data points. 
TABLE 3

Clean Parameters

\begin{tabular}{|c|c|c|c|c|c|c|c|}
\hline Filter & $L_{1}$ & $k\left(=r_{2} / r_{1}\right)$ & $r_{1}$ & $i(\mathrm{deg})$ & $L_{2}$ & $q\left(m_{2} / m_{1}\right)$ & $x^{2}$ \\
\hline \multicolumn{8}{|l|}{ 1988: } \\
\hline$V \ldots$ & $0.980 \pm 0.005$ & $0.611 \pm 0.012$ & $0.244 \pm 0.009$ & $89.0 \pm 7.9$ & $0.020 \pm 0.641$ & $0.531 \pm 0.097$ & 206.3 \\
\hline \multicolumn{8}{|l|}{ 1990: } \\
\hline B... & $0.962 \pm 0.003$ & $0.612 \pm 0.022$ & $0.235 \pm 0.009$ & $90.0 \pm 2.8$ & $0.016 \pm 0.005$ & $0.387 \pm 0.075$ & 138.3 \\
\hline$V$. & $0.965 \pm 0.005$ & $0.619 \pm 0.044$ & $0.229 \pm 0.008$ & $87.3 \pm 3.0$ & $0.031 \pm 0.006$ & $0.374 \pm 0.095$ & 53.5 \\
\hline$R$. & $0.961 \pm 0.004$ & $0.607 \pm 0.015$ & $0.236 \pm 0.006$ & $90.0 \pm 1.8$ & $0.041 \pm 0.005$ & $0.431 \pm 0.090$ & 33.8 \\
\hline$I \ldots$ & $0.918 \pm 0.004$ & $0.616 \pm 0.013$ & $0.238 \pm 0.005$ & $90.0 \pm 1.4$ & $0.072 \pm 0.005$ & $0.430 \pm 0.108$ & 57.5 \\
\hline \multicolumn{8}{|l|}{ 1991: } \\
\hline B ... & $0.959 \pm 0.003$ & $0.613 \pm 0.003$ & $0.247 \pm 0.002$ & $90.0 \pm 0.8$ & $0.007 \pm 0.004$ & $0.326 \pm 0.033$ & 521.6 \\
\hline$V \ldots \ldots$ & $0.959 \pm 0.003$ & $0.618 \pm 0.003$ & $0.244 \pm 0.002$ & $90.0 \pm 0.7$ & $0.018 \pm 0.004$ & $0.332 \pm 0.042$ & 366.3 \\
\hline$R \ldots \ldots$ & $0.949 \pm 0.003$ & $0.619 \pm 0.003$ & $0.241 \pm 0.002$ & $90.0 \pm 0.7$ & $0.034 \pm 0.004$ & $0.343 \pm 0.055$ & 299.1 \\
\hline I... & $0.900+0.003$ & $0.607+0.003$ & $0.240+0.002$ & $90.0+0.8$ & $0.059+0.004$ & $0.357+0.072$ & 304.6 \\
\hline \multicolumn{8}{|l|}{ 1992: } \\
\hline B ... & $0.965 \pm 0.006$ & $0.651 \pm 0.177$ & $0.212 \pm 0.029$ & 90 (fixed) & $0.018 \pm 0.008$ & $0.315 \pm 0.176$ & 51.9 \\
\hline$V \ldots \ldots$ & $0.985 \pm 0.005$ & $0.634 \pm 0.098$ & $0.222 \pm 0.016$ & 90 (fixed) & $0.031 \pm 0.007$ & $0.448 \pm 0.147$ & 27.5 \\
\hline$R$. & $0.941 \pm 0.005$ & $0.608 \pm 0.072$ & $0.230 \pm 0.012$ & 90 (fixed) & $0.042 \pm 0.006$ & $0.433 \pm 0.136$ & 26.5 \\
\hline$I$ & $0.913 \pm 0.005$ & $0.574 \pm 0.055$ & $0.232 \pm 0.009$ & 90 (fixed) & $0.071 \pm 0.006$ & $0.541 \pm 0.141$ & 32.0 \\
\hline \multicolumn{8}{|l|}{ 1994: } \\
\hline B.... & $0.964 \pm 0.004$ & $0.638 \pm 0.004$ & $0.245 \pm 0.003$ & $90.0 \pm 0.8$ & $0.019 \pm 0.005$ & $0.389 \pm 0.048$ & 70.5 \\
\hline$V \ldots \ldots$ & $0.973 \pm 0.004$ & $0.630 \pm 0.004$ & $0.242 \pm 0.003$ & $90.0 \pm 0.9$ & $0.020 \pm 0.005$ & $0.417 \pm 0.058$ & 41.8 \\
\hline$R \ldots \ldots$ & $0.936 \pm 0.005$ & $0.628 \pm 0.005$ & $0.240 \pm 0.003$ & $90.0 \pm 1.0$ & $0.037 \pm 0.006$ & $0.476 \pm 0.075$ & 49.5 \\
\hline$I \ldots$ & $0.924 \pm 0.005$ & $0.618 \pm 0.005$ & $0.241 \pm 0.003$ & $90.0 \pm 1.1$ & $0.061 \pm 0.006$ & $0.444 \pm 0.089$ & 52.2 \\
\hline \multicolumn{8}{|l|}{ 1995: } \\
\hline B $\ldots$ & $0.989 \pm 0.004$ & $0.626 \pm 0.003$ & $0.249 \pm 0.003$ & $90.0 \pm 1.2$ & $0.002 \pm 0.005$ & $0.377 \pm 0.046$ & 42.8 \\
\hline$V \ldots \ldots$ & $0.975 \pm 0.004$ & $0.624 \pm 0.004$ & $0.247 \pm 0.003$ & $90.0 \pm 1.2$ & $0.019 \pm 0.005$ & $0.391 \pm 0.059$ & 34.1 \\
\hline$R$. & $0.956 \pm 0.004$ & $0.622 \pm 0.004$ & $0.244 \pm 0.003$ & $90.0 \pm 1.5$ & $0.033 \pm 0.006$ & $0.415 \pm 0.074$ & 30.6 \\
\hline$I \ldots$ & $0.924 \pm 0.004$ & $0.614 \pm 0.004$ & $0.241 \pm 0.003$ & $90.0 \pm 1.5$ & $0.060 \pm 0.006$ & $0.546 \pm 0.091$ & 36.1 \\
\hline \multicolumn{8}{|l|}{ 1996: } \\
\hline B ...... & $0.966 \pm 0.006$ & $0.655 \pm 0.005$ & $0.247 \pm 0.005$ & $90.0 \pm 1.7$ & $0.024 \pm 0.007$ & $0.351 \pm 0.063$ & 127.4 \\
\hline$V \ldots \ldots$ & $0.957 \pm 0.006$ & $0.650 \pm 0.006$ & $0.250 \pm 0.006$ & $90.0 \pm 2.4$ & $0.027 \pm 0.008$ & $0.321 \pm 0.079$ & 65.3 \\
\hline$R \ldots \ldots$ & $0.937 \pm 0.007$ & $0.650 \pm 0.006$ & $0.248 \pm 0.073$ & $90.0 \pm 4.2$ & $0.043 \pm 0.008$ & $0.360 \pm 0.100$ & 57.6 \\
\hline$I \ldots \ldots$ & $0.892 \pm 0.007$ & $0.648 \pm 0.007$ & $0.242 \pm 0.005$ & $90.0 \pm 5.2$ & $0.083 \pm 0.009$ & $0.352 \pm 0.156$ & 52.3 \\
\hline \multicolumn{8}{|l|}{ 1997: } \\
\hline$V \ldots$ & $0.990+0.005$ & $0.606+0.004$ & $0.251+0.002$ & $90.0+2.2$ & $-0.004+0.006$ & $0.359+0.049$ & 79.9 \\
\hline$R \ldots \ldots$ & $0.967 \pm 0.005$ & $0.609 \pm 0.005$ & $0.249 \pm 0.003$ & $90.0 \pm 2.1$ & $0.019 \pm 0.006$ & $0.365 \pm 0.065$ & 53.1 \\
\hline$I \ldots \ldots$ & $0.931 \pm 0.005$ & $0.606 \pm 0.004$ & $0.247 \pm 0.003$ & $90.0 \pm 1.7$ & $0.051 \pm 0.006$ & $0.312 \pm 0.091$ & 38.1 \\
\hline
\end{tabular}

$270^{\circ}$ ALB tend to be at high latitudes. The majority of the spots modeled by Zeilik et al. (1990) were in the $90^{\circ}$ ALB, hence their observation of low-latitude spots. We have no explanation for this effect. Is it real, another statistical fluctuation, or a phase in a longer cycle?

Is there a latitudinal spot cycle? We observe a high percentage of high-latitude spots from 1990 until 1996.

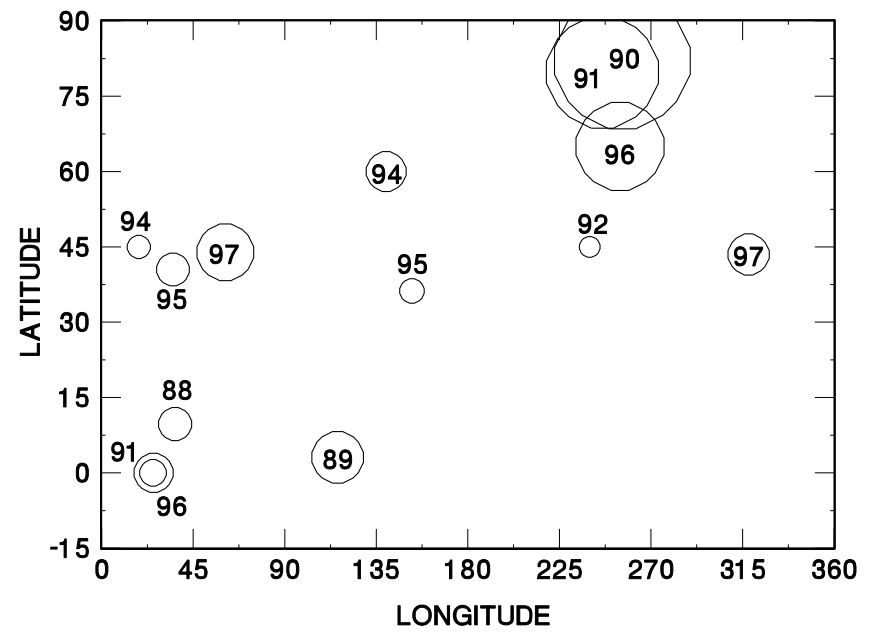

Fig. 19.-Mercator projection showing the locations and sizes of the modeled spots for our light curves. The numbers at each spot location represent the year in the 20th century that the spot was at that location.
Between 1964 and 1988, Zeilik et al. (1990) found mostly low-latitude spots. The one exception was in 1969, which was the only epoch observed between 1964 and 1973. If there were high-latitude spots during this decade, and if the 1990s are another era with high-latitude spots, then there might be a latitudinal drift cycle of between two and three decades. Systematic long-term observations will test this hypothesis.

Zeilik et al. (1990) were unable to obtain information about spot lifetimes or cycles because the light curves they analyzed were too intermittent. In this work, we begin to rectify that situation with annual light curves.

We usually obtained data in some combination of January, March, or May of each year, as can be seen from our observation log in Table 1 . We note several trends. Generally, we were unable to detect significant changes in the light curves over intervals of a few months. The year 1995 is a notable exception to this trend, as discussed above. We also note that there were smaller differences between the 1990 January and May data. The 1990 models incorporate only the more complete May light curve. As indicated in Table 1, if we detected no changes, we combined the data to model spot parameters. This relative stability in the light curves indicates that the spots on WY Cnc are typically stable for timescales of a few months, but can occasionally change rapidly. By contrast, other members of the shortperiod RS CVn group can show frequent changes in spot behavior over short time intervals, of a month or so (Zeilik 
et al. 1994; Heckert \& Zeilik 1991; Hempelmann et al. 1997). There are apparently differences in the short-term stability of spots in the short-period RS CVn group.

To try to understand why WY Cnc seems to have more stable spots and some other stars in this group have rapidly changing spots, we examined various properties of these systems, looking for correlations. The only trend we found was that the systems whose spots change rapidly tend to have higher X-ray fluxes as compiled by Strassmeier et al. (1993). Both WY Cnc and BH Vir have weak X-ray emission; only upper limits are listed. The X-rays indicate a high level of coronal activity. Both the high coronal activity levels and the rapid spot changes are driven by a high level of magnetic activity. Perhaps these stars go through longterm cycles. During the active phase of the cycle, spots change quickly, and during the quiescent phase, the spots are more stable. WY Cnc would then be in a quiescent phase while other group members are in an active phase. If this idea is correct, the relative number of stars in each phase suggests that the active phase would be longer than the quiescent phase. Does the rapid change we noticed in 1995 signal the onset of a more active phase during which the spots will change more rapidly?

Over the longer term (a few years), comparing spots for different years shows that the spots can be relatively stable over a period of 2-3 years. The spots appear to drift within the ALB, primarily toward increasing longitude. SV Cam also shows similar longitudinal drifts (Zeilik et al. 1988), which are on shorter timescales because SV Cam has more rapidly evolving spots. We currently have no evidence for significant latitudinal drifts in individual spots; however, there may be latitudinal drifts in active regions, as discussed above. After 2-3 years the spot apparently disappears and re-forms in the other ALB. We also note that in more recent years the individual spots seem less stable and are different each year. Continued systematic photometry is needed to pin down the spot cycles. Many unanswered questions remain. Is there a relationship between the spot size and lifetime? Do spots alternate ALBs? Are there any cycles in the long-term spot activity?

Zeilik et al. (1990) discussed the need for better estimates of the mass ratio, $q=M_{2} / M_{1}$, for the WY Cnc system. Normally, spectroscopy provides better mass information than photometry, but spectroscopy will not provide all the mass information for a single-lined binary. Hence they used the best light curves to find an average value of $q=0.356 \pm 0.176$. Using both their values and our new data (see Tables 2 and 3), we find an average value of $q=0.384 \pm 0.099$. The new value agrees well with the previous value and has a smaller error estimate. We can estimate absolute masses and radii using the values from our clean fits. Averaging our values and those of Zeilik et al. (1990), we find $k=r_{2} / r_{1}=0.625 \pm 0.034$ and $r_{1}=0.241$ \pm 0.008 . Following the method of Budding \& Zeilik (1987) we apply Kepler's third law and the mass-luminosity relation. We obtain $M_{1}=0.81, M_{2}=0.31, R_{1}=0.93$, and $R_{2}=0.58$, in solar units. These values agree well with the values derived by Zeilik et al. (1990). However, as they note, the value of $M_{2}$ derived from the mass function of Awadalla \& Budding (1979) is about 0.5 . We also note that Rao et al. (1991) also obtained a value of about 0.5 for $M_{2}$. However, their value appears to be the average value for the assumed spectral type of the secondary star, which they in turn used to compute other absolute system parameters.

\section{CONCLUSIONS}

WY Cancri has secular variations in the mean brightness levels out of eclipse and during the secondary eclipse that are not explained by spot models and indicate luminosity changes in the primary star. The spots on WY Cnc are relatively stable compared with other short-period RS CVn systems. The spots are often relatively stable for periods of months to a couple years. They can, however, change quickly on rare occasions.

The ALB at $270^{\circ}$ noted in previous work seems to confine spots well, but the $90^{\circ}$ ALB does not. A few low-latitude spots are outside the $90^{\circ}$ ALB. Contrary to previously noted trends to low-latitude spots, the spots on WY Cnc span the entire range of latitudes. The $270^{\circ}$ ALB tends to have high-latitude spots, and the spots in or near the $90^{\circ}$ ALB tend to be at low latitudes. Our clean fit results and derived absolute parameters agree well with previous work.

This work was supported by a Cottrell College science grant from the Research Corporation to P. A. H. During 1995 P. A. H. received support from the AAS Small Grants Program and from Western Carolina University in the form of both matching funds and from the Scholarly Development Assignment Program. J. I. O. received support from the AAS Research Experiences for Undergraduates program during 1994. M. A. H. received support from a Sigma Xi grant. We thank Ron Angione for scheduling the very generous amounts of telescope time needed for this long-term project. Publication costs were supported by a grant from NASA administered by the AAS.
Awadalla, N. S., \& Budding, E. 1979, Ap\&SS, 63, 479

Budding, E., \& Zeilik, M. 1987, ApJ, 319, 827

Hall, D. S. 1976, in IAU Colloq. 29, Multiple Periodic Variable Stars, ed. W. S. Fitch (Dordrecht: Reidel), 278

Hatzes, A. P., Vogt, S. S., Ramseyer, T. F., \& Misch, A. 1996, ApJ, 469, 808 Heckert, P. A., \& Zeilik, M. 1991, Inf. Bull. Variable Stars, No. 3636 . 1990, Inf. Bull. Variable Stars, No. 3416

Hempelmann, A., Hatzes, A. P., Kurster, M., \& Patkos, L. 1997, A\&A, 317, 125

Maceroni, C., Bianchini, A., Rodonò, M., Van 't Veer, F., \& Vio, R. 1990, A\&A, 237, 395

Rao, P. V., Sarma, M. B. K., \& Rao, B. V. N. S. P. 1991, J. Astrophys. Astron., 12, 225

\section{REFERENCES}

Sarma, M. K. R. 1976, Bull. Astron. Inst. Czechoslovakia, 27, 335

Schmidt, E. G. 1992, Inf. Bull. Variable Stars, No. 3733

Strassmeier, K., Hall, D. S., Fekel, F. C., \& Schenk, M. 1993, A\&AS, 100, 173

Thomas, J. H. 1979, Nature, 280, 662

Warner, B. 1988, Nature, 336, 129

Zeilik, M., Cox, D. A., Ledlow, M. J., Rhodes, M., Heckert, P. A., \& Budding, E. 1990, ApJ, 363, 647

Zeilik, M., De Blasi, C., Rhodes, M., \& Budding, E. 1988, ApJ, 332, 293

Zeilik, M., Gordon, S., Jaderlund, E., Ledlow, M. J., Summers, D. L., Heckert, P. A., Budding, E., \& Banks, T. S. 1994, ApJ, 421, 303 